\title{
MANEJO DE AZEVÉM RESISTENTE AO GLYPHOSATE EM POMARES DE MAÇÃ COM HERBICIDA SELECT (CLETHODIM)
}

\author{
Leandro Vargas; Erivelton Scherer Roman'; Mauro Antonio Rizzardi2; Roberto \\ Estêvão Bragion de Toledo ${ }^{3}$
}

\author{
1Eng.-Agr., Doutor, Pesquisador da Embrapa Trigo, Caixa Postal 451, CEP 99001-970 Passo Fundo, RS. \\ vargas@cnpt.embrapa.br \\ ${ }^{2}$ Eng.-Agr ${ }^{\circ}$., Doutor, Professor da Faculdade de Agronomia e Medicina Veterinária da Universidade de Passo \\ Fundo (UPF) . Passo Fundo, RS 99001-970 rizzardi@upf.tche.br \\ ${ }^{3}$ Eng.-Agr ${ }^{\circ}$., Doutor, Arysta LifeScience-Brasil- São Paulo, SP. roberto.toledo@arystalifescience.com
}

RESUMO - O glyphosate é um herbicida de amplo espectro, utilizado há mais de 15 anos, em pomares de maçã na região de Vacaria-RS, para manejo da vegetação nas linhas da cultura. São realizadas, em geral, três a quatro aplicações por ciclo e a dose normalmente utilizada é de 720 a $1080 \mathrm{~g}$ e.a. ha ${ }^{-1}$ de glyphosate (2 a $3 \mathrm{~L} \mathrm{ha}^{-1}$ do produto comercial). Azevém (Lolium multiflorum L.) é uma planta daninha comum em pomares e, tradicionalmente, sensível ao glyphosate. Os biótipos resistentes ao glyphosate não manifestam sintomas significativos de toxicidade em resposta a tratamentos com o herbicida glyphosate. O clethodim é um herbicida graminicida com alta eficiência de controle do azevém. O objetivo deste trabalho foi avaliar a eficiência do herbicida Select (clethodim) sobre os biótipos de azevém resistentes ao glyphosate. Para isso foi conduzido experimento a campo no ano de 2004, avaliando-se a resposta de azevém resistente a diferentes doses do herbicida Select. Os resultados evidenciam que a menor dose testada do herbicida $60 \mathrm{~g} \mathrm{ha}^{-1}$ de clethodim (Select, 0,25 $\mathrm{L} \mathrm{ha}^{-1}$ ) apresentou controle de 99\%, enquanto as doses acima desta $\left(0,3\right.$ e $\left.0,35 \mathrm{~L} \mathrm{ha}^{-1}\right)$ proporcionaram controle total (100\%) dos biótipos resistentes.

Palavras-chave: Lolium multiflorum; resistência; inibidores EPSPs; glyphosate.

\begin{abstract}
Glyphosate is a broad spectrum herbicide, used for more than 15 years in apple orchards in the Vacaria-RS region for vegetation management on the crops row. In general, 3-4 glyphosate applications are done in each crop cycle with the normal herbicide rate of $720-1,080 \mathrm{~g}$ e.a. ha ${ }^{-1}$. Lolium multiflorum is a frequently found weed specie in orchards and regularly sensible to glyphosate. Biotypes of Lolium multiflorum resistant to the glyphosate did not show any significant glyphosate symptoms. Clethodim is a highly efficient grass herbicide for Lolium multiflorum control. An experiment was carried out under field conditions during 2004 growing season in order to study the effects of herbicide Select (clethodim) on a ryegrass glyphosate resistant biotypes. The results indicated that the lower tested rate (Select $0,25 \mathrm{~L} \mathrm{ha}^{-1}$ or clethodim $\left.60 \mathrm{~g} \mathrm{ha}^{-1}\right)$ gave $99 \%$ control while the higher rates $(0,30$ and $0,35 \mathrm{~L} \mathrm{ha}^{-1}$ ) gave $100 \%$ control.
\end{abstract}

Key-words: Lolium multiflorum; resistance; EPSPs inhibitors; glyphosate. 


\section{INTRODUÇÃO}

O glyphosate é um herbicida de amplo espectro, utilizado há mais de 15 anos em pomares de maçã, na região de Vacaria-RS, para manejo da vegetação nas linhas da cultura. Em pomares, de forma geral, são realizadas entre três e quatro aplicações de produtos a base de glyphosate por ciclo, e a dose normalmente utilizada está entre 720 e $1080 \mathrm{~g}$ e.a. ha ${ }^{-1}$ de glyphosate $\left(2\right.$ a $3 \mathrm{~L} \mathrm{ha}^{-1}$ do produto comercial).

Azevém (Lolium multiflorum L.) é uma espécie anual de inverno que se caracteriza como a planta daninha mais comum em pomares de maçã na região de Vacaria-RS e é tipicamente sensível ao glyphosate. Entretanto, Roman et al. (2004) identificaram biótipos de azevém resistentes ao herbicida glyphosate. Os biótipos resistentes ao glyphosate não manifestam sintomas significativos de toxicidade em resposta a tratamentos com este herbicida. Assim, a ocorrência de plantas de azevém que, após receber o tratamento com glyphosate, não manifestam sintomas significativos de toxicidade, é comum em pomares de maçã e está aumentando em áreas cultivadas com outras culturas, como uva.

A resistência é a capacidade adquirida de uma planta em sobreviver a determinados tratamentos herbicidas que, sob condições normais, controlam os integrantes da população. O uso repetido de uma molécula herbicida pode selecionar biótipos resistentes de plantas daninhas preexistentes na população, levando ao aumento do seu número (Powles \& Holtum, 1994).

O número de espécies resistentes ao glyphosate identificadas está aumentando, sendo que, atualmente, são reconhecidas seis espécies resistentes em 20 diferentes regiões (Weed Science, 2003). O primeiro caso de Lolium multiflorum resistente ao glyphosate foi relatado por Perez \& Kogan (2002). O biótipo resistente foi identificado em pomares no Chile, que vinham recebendo, em média, três a quatro aplicações de glyphosate por ciclo durante, os últimos oito a 10 anos (Perez \& Kogan, 2002). Após 15 anos de uso bem sucedido de glyphosate na Austrália foram identificados biótipos de Lolium rigidum resistentes a este herbicida (Powles et al., 1998). Os biótipos resistem a doses sete a 11 vezes maiores de glyphosate do que os biótipos considerados sensíveis e apresentam-se suscetíveis a herbicidas com outros mecanismos de ação como paraquat, sethoxydim, simazine e fluazifop (Powles et al., 1998).

O Select (clethodim) é um herbicida graminicida que age inibindo a enzima ACCase, com alta eficiência, com controle acima de 95\%, sobre azevém, nos estádios de crescimento pós-inicial e pós-tardio (Lorenzi, 2000). Entretanto, não se conhece a resposta ao herbicida Select dos biótipos de azevém resistentes ao glyphosate. Assim, o objetivo deste trabalho foi avaliar a eficiência do herbicida clethodim (Select) sobre os biótipos de azevém resistentes a glyphosate.

\section{MATERIAL E MÉTODOS}

O experimento foi conduzido em condições de campo, em pomar de maçã em Vacaria/RS, durante os meses de outubro de 2004 a fevereiro de 2005.

Os tratamentos herbicidas foram: a) Roundup (1,5 $\left.\mathrm{L} \mathrm{ha}^{-1}\right)$; b) Roundup (3 L ha $\left.{ }^{-1}\right)$; c) Roundup (6 $\mathrm{L}$ ha $\left.{ }^{-1}\right)$; d) Roundup (12 $\left.\mathrm{L} \mathrm{ha}^{-1}\right)$; e) Roundup $\left(1,5 \mathrm{~L} \mathrm{ha}^{-1}\right)+$ 
Select $240 \mathrm{CE}\left(0,35 \mathrm{~L} \mathrm{ha}^{-1}\right)+\operatorname{Lanzar}\left(1 \mathrm{~L} \mathrm{ha}^{-1}\right)$; f) Select $240 \mathrm{CE}\left(0,35 \mathrm{~L} \mathrm{ha}^{-1}\right)+$ Lanzar ( $\left.\left.1 \mathrm{~L} \mathrm{ha}^{-1}\right) ; \mathrm{g}\right)$ Select $\left.240 \mathrm{CE}\left(0,3 \mathrm{~L} \mathrm{ha}^{-1}\right)+\operatorname{Lanzar}\left(1 \mathrm{~L} \mathrm{ha}^{-1}\right) ; \mathrm{h}\right)$ Select $240 \mathrm{CE}$ $\left(0,25 \mathrm{~L} \mathrm{ha}^{-1}\right)+\operatorname{Lanzar}\left(1 \mathrm{~L} \mathrm{ha}^{-1}\right)$; i) Select $240 \mathrm{CE}\left(0,125 \mathrm{~L} \mathrm{ha}^{-1}\right)+\operatorname{Targa}\left(0,6 \mathrm{~L} \mathrm{ha}^{-1}\right)+$ Lanzar (1 $\left.\mathrm{L} \mathrm{ha}^{-1}\right)$; j) testemunha sem aplicação de herbicida. O herbicida Roundup contém $360 \mathrm{~g} \mathrm{~L}^{-1}$ de glyphosate. $\mathrm{O}$ adjuvante Lanzar apresenta concentração de 280 $\mathrm{g} \mathrm{L}^{-1}$ e o herbicida Targa contém $50 \mathrm{~g} \mathrm{~L}^{-1}$ de quizalofop-p.

Os tratamentos foram aplicados quando as plantas de azevém estavam no estádio de três a quatro folhas, com aspersor costal de precisão, equipados com bicos XR 110.02, com volume de calda de $200 \mathrm{~L} \mathrm{ha}^{-1}$. O delineamento experimental usado foi completamente casualizado, com quatro repetições. A toxicidade dos tratamentos herbicidas foi avaliada utilizando-se escala percentual, em que nota zero significou nenhum efeito de dano às plantas e nota 100 representou morte ou completa supressão das mesmas. A toxicidade dos tratamentos herbicidas foi avaliada aos 7, 14, 21, 28 e 35 DAT (dias após tratamento).

Os dados obtidos foram submetidos à análise de variância e as médias comparadas entre si pelo teste de Duncan a $5 \%$ de probabilidade.

\section{RESULTADOS E DISCUSSÃO}

Na primeira avaliação, realizada aos 7 DAT, observou-se que as doses de 1,5 e 3,0 L ha ${ }^{-1}$ de Roundup (540 e $1080 \mathrm{~g}$ e.a. ha ${ }^{-1}$ de glyphosate, respectivamente) produziram toxicidade inferior a 20\%, enquanto as doses de 6 e $12 \mathrm{~L} \mathrm{ha}^{-1}(2160 \mathrm{e}$ $4320 \mathrm{~g}$ e.a. ha ${ }^{-1}$ de glyphosate, respectivamente) produziram toxicidade de 38 e $53 \%$ (Tabela 1). O tratamento contendo $1,5 \mathrm{~L} \mathrm{ha}^{-1}$ de Roundup (540 g e.a. ha ${ }^{-1}$ de glyphosate) mais $0,35 \mathrm{~L} \mathrm{ha}^{-1}$ de Select (84 $\mathrm{g} \mathrm{ha}^{-1}$ de clethodim) mais $1,0 \mathrm{~L} \mathrm{ha}^{-1} \mathrm{de}$ Lanzar (280 $\mathrm{g} \mathrm{ha}^{-1}$ ) provocou toxicidade de $28 \%$, ou seja, apresentou toxicidade superior a dose de 1,5 L ha ${ }^{-1}$ de Roundup (8,7\% de toxicidade) e 0,35 $\mathrm{L} \mathrm{ha}^{-1}$ de Select (12,5\% de toxicidade), aplicados de forma isolada (Tabela 1). Isso indica que houve efeito sinérgico da mistura, já que a toxicidade desta foi superior a soma das toxicidades proporcionadas pelos produtos quando aplicados de forma isolada. Os tratamentos contendo o herbicida Select, aos 7 DAT, apresentaram toxicidade inferior a $12,5 \%$, independentemente da dose avaliada (Tabela 1).

$\mathrm{Na}$ segunda avaliação, realizada aos 14 DAT, observou-se evolução nos sintomas de toxicidade para todos os tratamentos avaliados. Os tratamentos que apresentaram maior toxicidade, próximo de $80 \%$, foram Roundup $12 \mathrm{~L} \mathrm{ha}^{-1}$ (4320 g e.a. ha ${ }^{-1}$ de glyphosate), a associação de Roundup + Select + Lanzar, e o tratamento contendo Select + Targa (quizalofop-p) + Lanzar (Tabela 1). Os demais tratamentos apresentaram toxicidade próxima a $50 \%$, com exceção do tratamento com $1,5 \mathrm{~L} \mathrm{ha}^{-1}$ de Roundup (540 g e.a. ha $^{-1}$ de glyphosate), que foi de $12,5 \%$.

A terceira avaliação, realizada aos 21 DAT, evidenciou a estabilização dos sintomas de toxicidade provocados pelos tratamentos contendo Roundup (glyphosate) de forma isolada (Tabela 1). Já o tratamento composto por Roundup + Select + Lanzar e o tratamento contendo a maior dose de Select $\left(0,35 \mathrm{~L} \mathrm{ha}^{-1}\right)$ evidenciaram toxicidades semelhantes e superior a $95 \%$. Os tratamentos contendo as doses de $0,30 \mathrm{~L} \mathrm{ha}^{-1}$ e $0,25 \mathrm{~L} \mathrm{ha}^{-1}$ de Select (72 e $60 \mathrm{~g}$ i.a. ha ${ }^{-1}$ de clethodim), e aquele composto por Select + Targa + Lanzar apresentaram evolução nos sintomas, resultando em toxicidade acima de $83 \%$. 
$\mathrm{Na}$ quarta avaliação, realizada aos 28 DAT, observou-se recuperação das plantas tratadas com Roundup (glyphosate) de forma isolada, ou seja, os sintomas observados foram menores do que aqueles anotados na avaliação anterior, realizada aos 21 DAT (Tabela 1). Os demais tratamentos apresentaram toxicidade semelhante e acima de $90 \%$.

A última avaliação, realizada aos 35 DAT, confirmou o processo de recuperação das plantas tratadas com Roundup (glyphosate) de forma isolada, sendo que as doses de 1,5 e de 3,0 $\mathrm{L} \mathrm{ha}^{-1}$ de Roundup apresentaram toxicidade de apenas 6,2 e 11,2\%, respectivamente. Já as doses de 6 e a de $12 \mathrm{~L} \mathrm{ha}^{-1}$ apresentaram toxicidade de 35 e 63\%, respectivamente (Tabela 1). Os demais tratamentos apresentaram controle total da população de plantas de azevém resistentes ao glyphosate, com exceção do tratamento com Select na dose de 0,25 L $\mathrm{ha}^{-1}$ (60 $\mathrm{g} \mathrm{ha}^{-1}$ de clethodim), que proporcionou toxicidade de 99\% (Tabela 1).

De maneira geral observou-se que o tratamento composto por Roundup (glyphosate) + Select (clethodim) + Lanzar, apresentou sintomas de toxicidade acima de $80 \%$ já aos 14 DAT. Entretanto, nas avaliações realizadas após os 14 DAT não houve diferença entre este tratamento e aquele com Select (clethodim) + Lanzar, na dose de $84 \mathrm{~g} \mathrm{ha}^{-1}$. Isso evidencia que a adição do glyphosate apenas antecipou a manifestação dos sintomas, mas não aumentou o nível de controle final. Especula-se que glyphosate poderá ter ação aditiva ou sinérgica quando em mistura com clethodim, contudo existe a necessidade de adequação da dose desse graminicida.

Além disso, observou-se que a evolução dos sintomas está relacionada com a dose empregada do herbicida Select (clethodim), ou seja, quanto maior a dose mais rápida foi a evolução dos sintomas. Contudo, aos 35 DAT todas as doses avaliadas do Select (clethodim) apresentaram toxicidade semelhante.

Os resultados permitem afirmar que as doses 0,$25 ; 0,30$ e $0,35 \mathrm{~L} \mathrm{ha}^{-1}$ de Select, (60; 72; e $84 \mathrm{~g}$ de clethodim, respectivamente), mais $1 \mathrm{~L} \mathrm{ha}^{-1}$ de Lanzar (280 $\left.\mathrm{g} \mathrm{ha}^{-1}\right)$, controlam eficientemente a população de azevém resistente; que doses de até $12 \mathrm{~L} \mathrm{ha}{ }^{-1}$ de Roundup (4320 g e.a. ha ${ }^{-1}$ de glyphosate) não apresentam controle satisfatório da população de azevém resistente; que a associação do herbicida Select com Roundup (glyphosate) ou com Targa (quizalofop-p) não proporcionou aumento no nível final de controle da população de azevém resistente em comparação com as doses de Select avaliadas de forma isolada.

\section{LITERATURA CITADA}

LORENZI, H. Manual de identificação e manejo de plantas daninhas plantio direto e convencional. 5 ed. Nova odessa, SP: Instituto Plantarum. 2000. 339p.

PEREZ, A.; KOGAN, M. Glyphosate-resistant Lolium multiflorum in Chilean orchards. Weed Research, v.43, p.12-19, 2002.

POWLES, S. B. \& HOLTUM, J. A. M. Herbicide resistance in plants: biology and biochemistry. Boca Raton, 1994.

POWLES, S.B.; LORRAINE-COLWILL, D.F., DELLOW, J.J.; PRESTON, C. Evolved resistance to glyphosate in rigid ryegrass (Lolium rigidum) in Australia. Weed Science, v. 46, p. 604-607,1998. 
ROMAN, E. S.; VARGAS, L.; RIZZARDI, M. A.; MATTEI, R.W. Resistência de azevém (Lolium multiflorum) ao herbicida glyphosate. Planta Daninha, Viçosa, v.22, n.2. p.301-306. 2004

WEED SCIENCE - Glycines (g/9) resistant weeds by species and country. http://www.weedscience.org/Summary/UspeciesMOA.asp?IstMOAID=12\&FmHRAC Group=Go. Consultado em 10 de fev. de 2003. 
Tabela 1 - Controle de azevém resistente a glyphosate com diferentes doses de Select (clethodim).

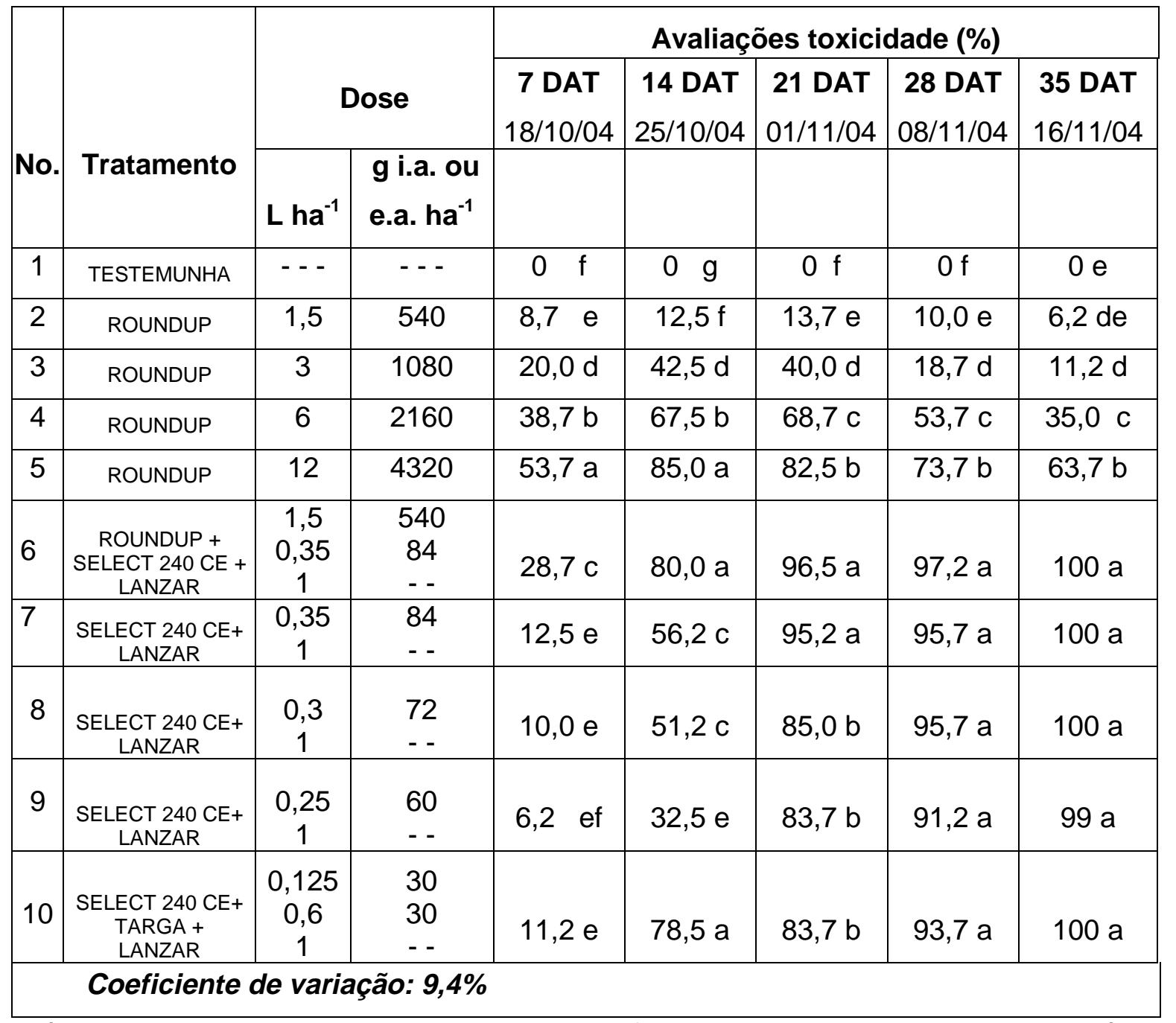

*Médias seguidas pela mesma letra, na coluna, não diferem entre si, pelo teste de Duncan ao nível de $5 \%$ de probabilidade. 